\title{
Ampere per Square Meter
}

National Cancer Institute

\section{Source}

National Cancer Institute. Ampere per Square Meter. NCI Thesaurus. Code C68908.

A SI derived unit of an electric current density which is equal to an electric current of one ampere circulating in a homogeneous conductor with a cross section area of one square meter. 